\title{
Some more properties of the bisect-diagonal quadrilateral
}

\author{
MICHAEL DE VILLIERS
}

Martin Josefsson [1] has coined the term 'bisect-diagonal quadrilateral' for a quadrilateral with at least one diagonal bisected by the other diagonal, and extensively explored some of its properties. This quadrilateral has also been called a 'bisecting quadrilateral' [2], a 'sloping-kite' or 'sliding-kite' [3], or 'slant kite' [4]. The purpose of this paper is to explore some more properties of this quadrilateral.

A familiar property of the bisect-diagonal quadrilateral that is proved in Coxeter [5, pp. 54-55) as well as in Josefsson [1, pp. 215], and is extended to the concave case by Pillay \& Pillay [6, pp. 16-17], is the following:

Theorem 1: A quadrilateral is a bisect-diagonal quadrilateral (where at least one diagonal bisects the other) if, and only if, the diagonal that bisects the other also bisects the area of the quadrilateral.

\section{Equipartitioning point of a quadrilateral}

As is well known, the centroid $G$ of a triangle $A B C$ divides, or equipartitions, the triangle into three triangles, $A G B, B C G$ and $C G A$, of equal area.

The question now arises whether one can find a similar point $P$ for a quadrilateral $A B C D$ that divides, or equi-partitions, it into four triangles, $A P B, B P C, C P D$ and $D P A$, of equal area. For a parallelogram, it's obvious that such an 'equipartitioning' point $P$ exists, and would be located at its centroid, i.e. the intersection of its diagonals. But what about a more general quadrilateral? Where can $P$ be located?

Based on the example of the triangle and the parallelogram, one may intuitively feel that in general such a point would be located at either the point mass centroid or the lamina centroid of a quadrilateral. However, a quick experimental check using an accurately constructed sketch with dynamic geometry as shown in Figure 1, shows that neither the point mass centroid $^{*} G_{P M}$ nor the lamina centroid ${ }^{\dagger} G_{L}$ respectively divide the quadrilaterals $A B C D$ and $K L M N$ into four triangles of equal area. Since $G_{L}$ is the balancing point of the lamina (cardboard) quadrilateral $A B C D$, one would have anticipated that the four triangles subtended by $G_{L}$ and the four sides would be equal in area. This not being the case in general as shown in Figure 1, therefore seems a bit counter-intuitive and unexpected.

\footnotetext{
The point mass centroid of a quadrilateral is located at the intersection of the lines connecting the midpoints of opposite sides.

$\doteqdot$ The lamina centroid of a quadrilateral is located at the intersection of the line connecting the centroids of triangles $K L M$ and $M N K$ with the line connecting the centroids of triangles $K L N$ and $L M N$.
} 
Area $\triangle A G_{p m} B=13.58 \mathrm{~cm}^{2}$

Area $\triangle B G_{p m} C=8.24 \mathrm{~cm}^{2}$

Area $\triangle C G_{p m} D=16.37 \mathrm{~cm}^{2}$

Area $\triangle D G_{p m} A=21.70 \mathrm{~cm}^{2}$

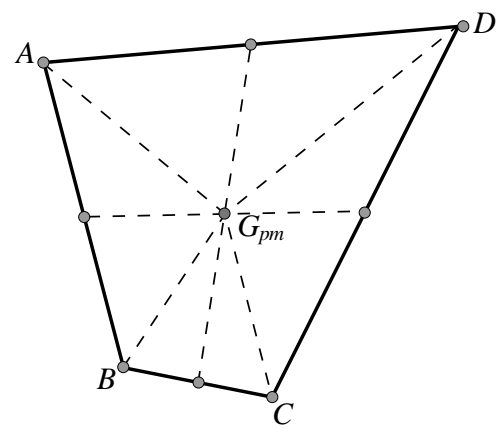

Area $\triangle K G_{L} L=15.18 \mathrm{~cm}^{2}$

Area $\triangle L G_{L} M=18.78 \mathrm{~cm}^{2}$

Area $\triangle M G_{L} N=22.86 \mathrm{~cm}^{2}$

Area $\triangle N G_{L} K=21.29 \mathrm{~cm}^{2}$

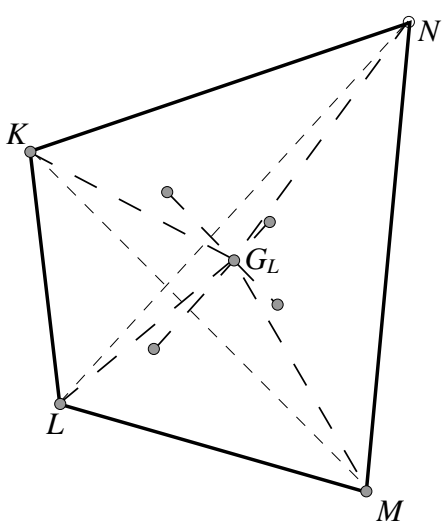

FIGURE $1 *$

The reader may now wish to use the following online dynamic sketch to explore experimentally where such a point $P$ might be located for a general quadrilateral or some special cases:

http://dynamicmathematicslearning.com/equipartitioning-quad.html

Quite remarkably, such a (equipartitioning) point $\mathrm{P}$ that divides, or equipartitions, a quadrilateral into four triangles of equal area exists only for a bisect-diagonal quadrilateral. This follows from the following little known theorem proved by Pillay \& Pillay [6] \& Gilbert et al. [7, pp. 68-70]:

Theorem 2: A quadrilateral has an equipartitioning point $P$ if, and only if, it is a bisect-diagonal quadrilateral, and then $P$ is the midpoint of the diagonal bisecting the other.

The proof that the midpoint of the diagonal bisecting the other is the equipartitioning point $P$ of a bisect-diagonal quadrilateral follows directly from Theorem 1, and is left to the reader. The following proof that only a bisect-diagonal quadrilateral has an equipartitioning point is slightly modified from that of [6] \& [7], and is given below only for the convex case.

On the previous page you have $G_{P M}$ and here $G_{p m}$. Seems strange. 


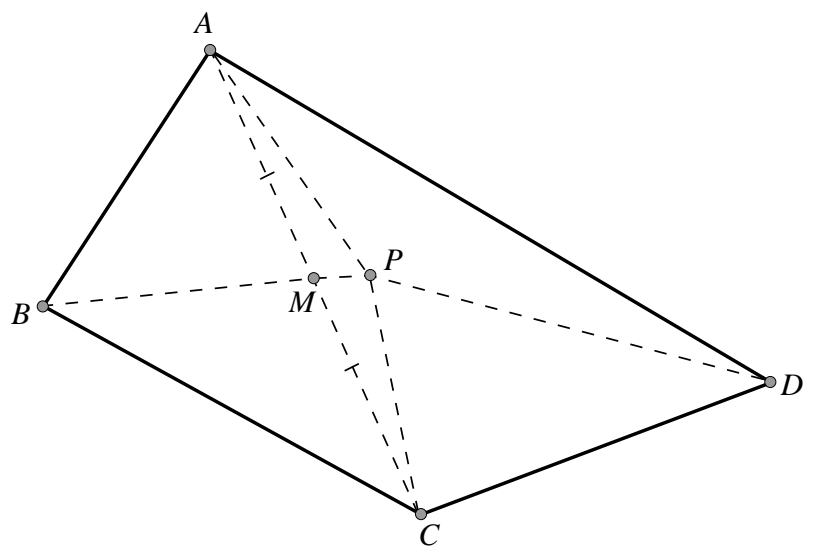

FIGURE 2

Proof: Suppose a convex quadrilateral $A B C D$ has an equipartitioning point $P$ as shown in Figure 2. Since it is given that triangles $A P B, B P C, C P D$ and $D P A$ are equal in area, it follows that diagonals $B P$ and $D P$ bisect the areas of quadrilaterals $A B C P$ and $A P C D$ respectively. Hence, from Theorem 1, both straight lines $B P$ and $D P$ extended contain the midpoint $M$ of $A C$.

This implies that $D P M$ is a straight line, and since the straight line through $M$ and $P$ must contain both $B$ and $D$ we conclude that $B M P D$ must coincide with the diagonal $B D$, and that $B D$ bisects $A C$ in $M$. But triangles $A P B$ and $D P A$ have the same area, so $B P=P D$. Thus we have shown that diagonal $B D$ bisects diagonal $A C$ and that the equipartitioning point $P$ is the midpoint of $D B$.

Of course, the argument is entirely exchangeable, and we could in the same way argue that diagonal $A C$ bisects diagonal $B D$ and that the equipartitioning point $P$ is the midpoint of $A C$. Either way, the result is proved that at least one of the diagonals of $A B C D$ is bisected by the other.

The same argument, with a few modifications, applies when quadrilateral $A B C D$ is concave, but is left to the reader. As shown in [7, pp. 69-70], one can also prove this theorem using a trigonometric argument that extends to the concave case.

\section{Lamina and point mass centroids of a bisect-diagonal quadrilateral}

Let us now examine the lamina and point mass centroids of a bisectdiagonal quadrilateral, and any relationship between them.

Given a bisect-diagonal quadrilateral $A B C D$ as shown in Figure 4 with $M$ the midpoint of the bisected diagonal $B D$ and $P$ the midpoint of diagonal $A C$. (According to Theorem 2, the point $P$ is therefore the equipartitioning point of $A B C D$.) 


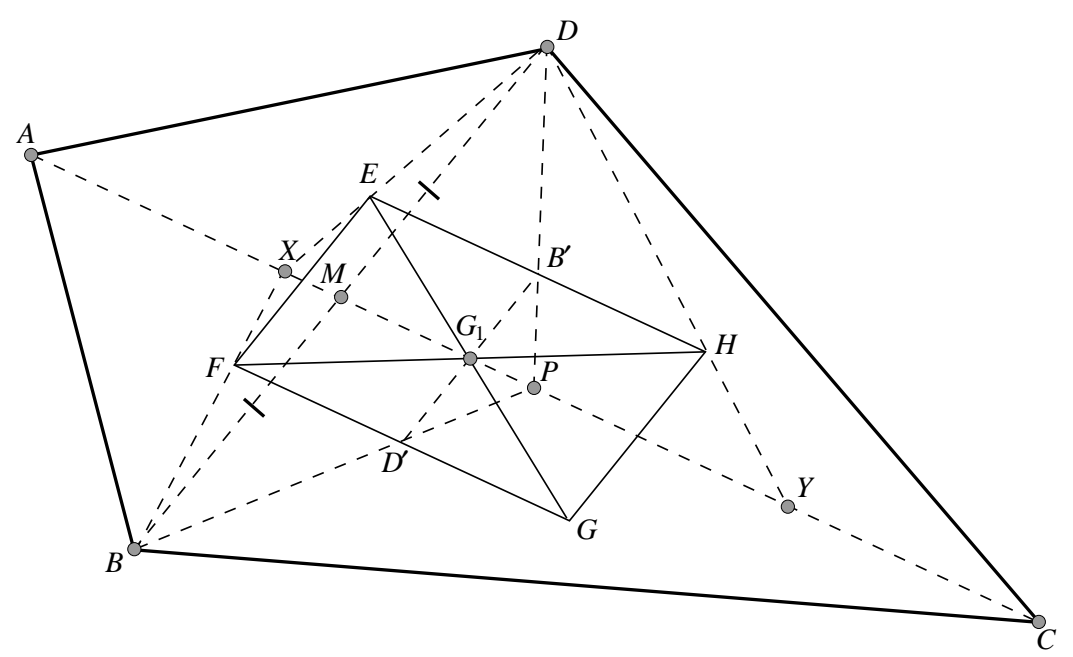

FIGURE 3

Theorem 3: Construct the centroids of triangles $D P A, A P B, B P C$ and $C P D$ of a bisect-diagonal quadrilateral $A B C D$ and label them respectively, $E, F, G$ and $H$. Then $E F G H$ is a parallelogram and the intersection of its diagonals, $G_{1}$, lies on $A C$, and is the lamina centroid of $A B C D$.

Proof: Since $E$ lies on the median $D X$ of triangle $D P A$ and $H$ lies on the median $D Y$ of triangle $C P D$, it follows that $E H / / X Y$ and $E H=\frac{2}{3} X Y$. Similarly, $F G / / X Y$ and $F G=\frac{2}{3} X Y$. Hence opposite sides $E H$ and $F G$ are parallel and equal, which shows that $E F G H$ is a parallelogram. Since the areas of triangles $D P A, A P B, B P C$ and $C P D$ are equal, the weight of their respective laminas are equally concentrated at their centroids; hence all together, their lamina weights balance at the intersection, $G_{1}$, of the diagonals of $E F G H$. Moreover, since $E H$ and $F G$ are the same distance away from $A C$, it follows that $A C$ passes through the symmetrical point, $G_{1}$, of $E F G H$. This completes the proof of Theorem 3.

In addition, since $X P=P Y$, note that $B^{\prime}$, the centroid of triangle $A C D$, is the midpoint of $E H$. Similarly, $D^{\prime}$ is the midpoint of $F G$. Since the centroids $A^{\prime}$ and $C^{\prime}$, respectively, of triangles $B C D$ and $A B D$, lie on diagonal $A C$, the line $B^{\prime} D^{\prime}$ also intersects the line $A^{\prime} C^{\prime}$ (line $A C$ ) at the lamina centroid, $G_{1}$. 


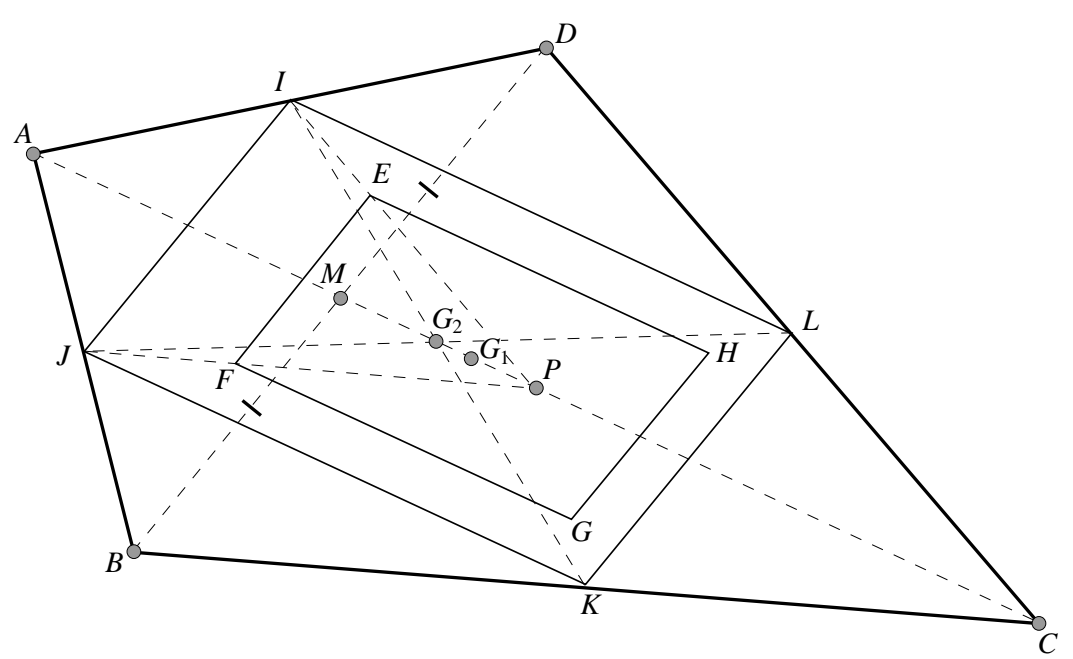

FIGURE 4

Theorem 4: The lamina parallelogram $E F G H$ of a bisect-diagonal quadrilateral $A B C D$ is homothetic to the Varignon parallelogram $I J K L$ formed by the midpoints of the sides of $A B C D$, with the centre of similarity between the two located at $P$, and a scale factor of $\frac{2}{3}$.

Proof: Since $E$ and $F$ are the respective centroids of triangles $D P A$ and $A P B$, we have in triangle $I P J$ that $E F / / I J$ and $E F=\frac{2}{3} I J$. Since the same can be shown for the other pairs of corresponding sides of $E F G H$ and $I J K L$, it follows that $E F G H$ is homothetic to $I J K L$ with centre $P$ and scale factor $\frac{2}{3}$.

Theorem 5: The distance between the lamina centroid $G_{1}$ and the equipartitioning point $P$ of a bisect-diagonal quadrilateral is twice that of the distance between its lamina centroid $G_{1}$ and point mass centroid $G_{2}$.

Proof: Since the point mass centroid $G_{2}$ is located at the intersection of the diagonals of the Varignon parallelogram $I J K L$, it follows from the similarity transformation in Theorem 4 that $G_{1} P=2 G_{2} G_{1}$.

In addition, according to a well-known result in [5, p. 54] and [1, p. 216] the point mass centroid $G_{2}$ also lies at the midpoint of the line segment $M P$. Hence $3 G_{2} G_{1}=G_{2} P \Rightarrow 6 G_{2} G_{1}=M P$.

\section{The Newton-Gauss line}

Since the celebrated Newton-Gauss line [8, p. 62] is the straight line containing the midpoints of the three diagonals of a complete quadrilateral, it immediately follows that the diagonal $A C$ passes through the midpoint $S$ of the third diagonal $Q R$ of the complete bisect-diagonal quadrilateral $A B C D Q R$. 


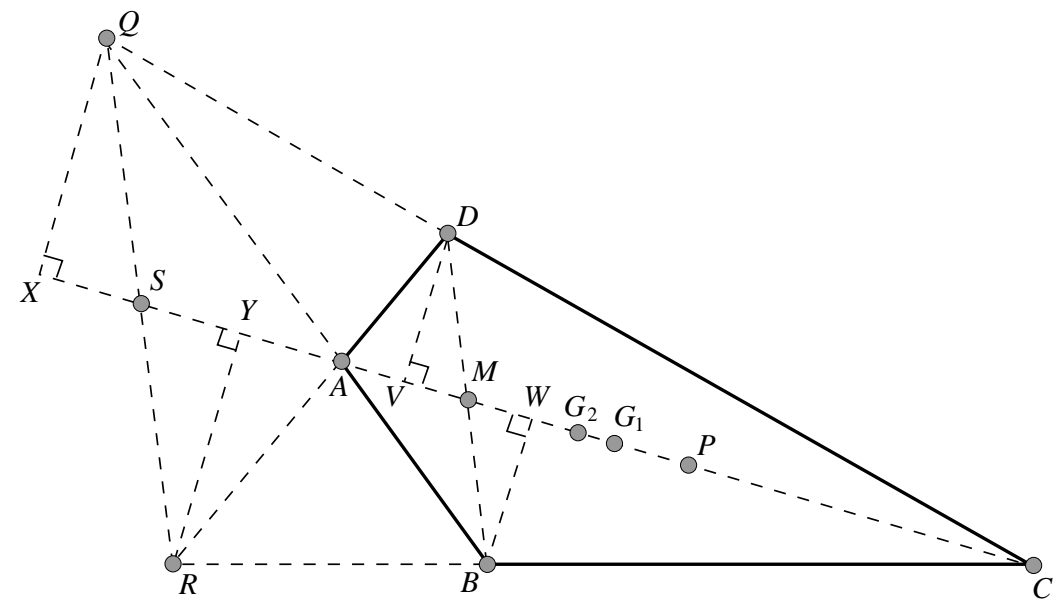

FIGURE 5

Theorem 6: Given a complete bisect-diagonal quadrilateral $A B C D Q R$ as shown in Figure 5 with diagonal $A C$ bisecting diagonal $B D$, then the third diagonal $Q R$ is parallel to $B D$.

Proof: Drop perpendiculars from $Q, R, D$ and $B$ to $A C$. From the similarity of triangles $Q X C$ and $D V C$ it follows that $\frac{C D}{C Q}=\frac{D V}{Q X}$. Similarly, $\frac{C B}{C R}=\frac{B W}{R Y}$. From the congruency of triangles $Q X S$ and $R Y S$, and of triangles $D V M$ and $B W N$, we have $\frac{D V}{Q X}=\frac{B W}{R Y}$. Hence $\frac{C D}{C Q}=\frac{C B}{C R}$, which implies that $Q R$ is parallel to $B D$.

Conversely, given a complete quadrilateral $A B C D Q R$ with diagonal $Q R$ parallel to $B D$, then it is easy to see that the above argument applies in reverse, and that diagonal $A C$ will bisect diagonal $B D$. In other words, $A B C D$ will be a bisect-diagonal quadrilateral.

\section{Concluding comment}

Apart from parallelograms and kites as special cases of a bisectdiagonal quadrilateral, it might also be of interest to some readers to note that that any cyclic quadrilateral $A B C D$ with its sides $A B: B C: C D: D A$ in geometric progression with common ratio $r$, as shown in [9], is also a bisectdiagonal quadrilateral. It is easy to establish and left as an exercise.

Note: A dynamic geometry sketch illustrating the properties of a bisectdiagonal quadrilateral explored here is available online at:

http://dynamicmathematicslearning.com/bisect-diagonal-quadrilateral.html 


\section{References}

1. M. Josefsson, Properties of bisect-diagonal quadrilaterals, Math. Gaz. 101 (July 2017) pp. 214-226.

2. M. de Villiers, Some adventures in Euclidean geometry, Dynamic Mathematics Learning (2009).

3. G. Graumann, Investigating and ordering quadrilaterals and their analogies in space - problem fields with various aspects, ZDM 37 (3) (2005) pp. 190-198,

also available at: http//subs.emis.de/journals/ZDM/zdm053a8.pdf

4. A. Ramachandran, The four-gon family tree, At Right Angles 1 (1) (2012) pp. 53-57, also available at http://www.teachersofindia.org/sites/ default/files/12_four_gon_family_tree.pdf

5. H. S. M. Coxeter \& S. L. Greitzer, Geometry revisited, Math. Ass. Amer. (1967).

6. S. Pillay \& P. Pillay, Equipartitioning and balancing points of polygons, Pythagoras, 71 (July 2010) pp. 13-21.

7. G. T. Gilbert, M. Krusemeyer and L. C. Larson, The Wohascum County problem book, Dolciani Mathematical Expositions, 14(10), Math. Ass. Amer. (1993).

8. R. A. Johnson, Advanced Euclidean geometry, Dover Publications (1960).

9. M. De Villiers, A cyclic Kepler quadrilateral \& the Golden Ratio, At Right Angles, March 2018, pp. 91-94. (Accessed on 16 August 2020 at: https://azimpremjiuniversity.edu.in/SitePages/resources-ara-march2018-cyclic-kepler-quadrilateral.aspx ).

MICHAEL DE VILLIERS Mathematics Education (RUMEUS), University of Stellenbosch, South Africa e-mail: profmd@mweb.co.za 